\title{
Mineração
}

\section{Um modelo de programação matemática para alocação estática de caminhões visando ao atendimento de metas de produção e qualidade}

\author{
Felippe Pereira da Costa \\ Mestrando -PPGEM/EM/UFOP - Ouro Preto-MG.E-mail: felippe@mineral.em.ufop.br
}

Marcone Jamilson Freitas Souza

Prof. Adjunto - PPGEM/EM/UFOP e DECOM/ICEB/UFOP - Ouro Preto - MG. E-mail: marcone@iceb.ufop.br

Luiz Ricardo Pinto

Prof. Adjunto-DEP/UFMG - Belo Horizonte-MG.E-mail: luiz@dep.ufmg.br

\section{Resumo}

Esse trabalho desenvolve um modelo de programação linear por metas (goal programming) aplicado ao planejamento de produção em mineração. O objetivo é determinar o ritmo de lavra de cada frente, considerando a alocação de equipamentos de carga e transporte, de modo a fornecer à usina de beneficiamento uma alimentação adequada. Como cada frente possui características de qualidade diferentes, o ritmo de lavra deve ser realizado de forma proporcional, sendo limitado pela capacidade de produção dos equipamentos de carga e transporte disponíveis na mina. A mistura do minério proveniente de cada frente deve gerar uma alimentação que atenda as metas de qualidade e produção conforme as especificações preestabelecidas. Nesse trabalho, foi adotado o sistema de alocação estática de caminhões, por ser geralmente utilizado em minerações de pequeno e médio porte, devido à simplificação das operações e ao alto custo de implantação de um sistema de despacho eletrônico de caminhões. O modelo de programação matemática desenvolvido foi implementado no software de otimização LINGO 7.0 interfaceando com planilhas do EXCEL 2000, proporcionando, dessa forma, uma melhor interação entre o usuário e o aplicativo. O modelo foi validado usando uma instância-teste disponível na literatura, acrescida de metas de produção e qualidade.

Palavras-chave: planejamento de lavra, mistura de minérios, programação matemática, alocação estática.

\begin{abstract}
This paper develops a goal programming model applied to mining production planning. The objective is to determine the run of each working bench, considering the allocation of load and haulage equipment, in order to supply the processing plant with an appropriate feeding. As each working bench holds different characteristics of quality, the run must be accomplished in a proportional way, being limited by the production capacity of the load and haulage equipment available in the mine. The blending of ore from each working bench should generate a feeding considering quality and production goals according to the given specifications. In this paper the truck static allocation system was adopted for being generally used by small and medium mines, due to the simplification of the operations and at the high cost of implantation of a truck dispatching system. The mathematical programming model was implemented using the optimization software LINGO 7.0 in conjunction with EXCEL 2000 spreadsheet, providing a better interaction between the user and the application. The model was validated using a test instance from the literature, added with production and quality goals.
\end{abstract}

Keywords: mining planning, ore blending, mathematical programming, static allocation system. 
Um modelo de programação matemática para alocação estática de caminhões...

\section{Introdução}

O principal objetivo do planejamento de produção em uma mina a céu aberto é a determinação de qual ritmo de lavra será implementado em cada frente, fornecendo ao cliente um produto adequado, seja o cliente a usina de beneficiamento (cliente interno) ou um cliente externo. Esse problema é conhecido na literatura como problema da mistura ou blendagem.

Em casos reais da indústria mineral, é necessário considerar uma série de outras questões, que, normalmente, não são abordadas em conjunto na literatura. Otimizar separadamente o problema sem contemplar essas questões pode gerar conflitos que inviabilizam a implementação das soluções. Uma dessas questões diz respeito ao atendimento às metas, abordada por Chanda e Dagdelen (1995), sejam elas de produção ou qualidade. $\mathrm{O}$ atendimento às metas de produção é importante, pois uma produção superior à requerida pode causar problemas como a falta de espaço adequado em estoque e custos adicionais de manuseio, já uma produção inferior causa uma redução na taxa de utilização dos equipamentos da mina e da usina de beneficiamento, além de multas contratuais pelo não fornecimento do produto. Outro aspecto de grande importância relacionado ao atendimento às metas, agora considerando as especificações de qualidade da mistura, está ligado, no caso da usina de beneficiamento, ao controle das flutuações que devem ser mínimas, tornando o processamento mais eficiente, ou, ainda, previamente determinadas para que sejam tomadas as devidas providências de ajuste na usina de beneficiamento.

Três outras questões relacionadas à realidade das operações em minas a céu aberto são abordadas por Pinto e Merschmann(2001), Merschmann (2002) e Pinto et al. (2003). A primeira refere-se à relação estéril/minério e visa a prevenir que somente minério seja lavrado, possibilitando a liberação de novas frentes de minério ou a implantação de obras necessárias para viabilizar as operações na mina. A segunda diz respeito à alocação de equipamentos de carga. A alocação de um equipamento de carga deve ser realizada de modo a utilizar ao máximo sua capacidade de produção, pois ela limita o ritmo de lavra da frente onde está alocada e deve, ainda, considerar a compatibilidade com os equipamentos de transporte. A terceira questão está relacionada ao sistema de alocação de equipamentos de transporte, no caso desse trabalho, o sistema de alocação estática de caminhões.

Esse trabalho desenvolve um modelo de programação linear por metas, contemplando todas essas questões levantadas anteriormente. Adicionalmente, reduz-se, substancialmente, o número de restrições do modelo proposto em Pinto e Merschmann (2001) e substituem-se as restrições não lineares propostas nesse trabalho por restrições lineares equivalentes, de forma a garantir a otimalidade da solução gerada. O modelo foi implementado no software de otimização LINGO 7.0 interfaceando com planilhas do EXCEL 2000, proporcionando, dessa forma, uma melhor interação entre o usuário e o aplicativo. $\mathrm{O}$ modelo foi validado usando uma instância-teste disponível em Pinto et al.(2003), acrescida de metas de produção e qualidade.

Esse trabalho está organizado como segue. Na seção 2, descreve-se o problema de alocação estática de caminhões. $\mathrm{Na}$ seção 3, apresenta-se o modelo de programação linear por metas para resolver o problema abordado. Os resultados obtidos pela aplicação do modelo são apresentados e discutidos na seção 4, enquanto a última seção conclui o trabalho.

\section{Descrição do problema de alocação estática de caminhões}

O problema de alocação estática de caminhões abordado engloba os problemas de mistura de minérios e de alocação de equipamentos de carga, os quais encontram-se descritos nas seções 2.1 e 2.2 .

\subsection{Mistura de minérios}

O problema da mistura de minérios considerado nesse trabalho consiste na determinação de quanto minério proveniente de cada frente deve ser misturado de modo a satisfazer as exigências do cliente. Como cada frente de lavra possui características de qualidade diferentes, tais como o teor de determinado elemento químico ou a percentagem de minério em determinada granulometria, torna-se necessário determinar a proporção do ritmo de lavra de cada frente que gere uma alimentação que atenda as metas de qualidade e produção preestabelecidas. Entretanto tais metas podem não ser alcançadas. Assim, para que o produto seja aceito, os parâmetros de qualidade devem ter valores pertencentes a um intervalo especificado pelo cliente. Vale lembrar que o termo "mistura de minérios" diz respeito à determinação dessa proporção e não deve ser confundido com "homogeneização de minério", processo no qual pretende-se que todo o minério misturado possua as mesmas características.

\subsection{Alocação de equipamentos de carga}

Para viabilizar a lavra em diferentes frentes, uma mina conta com uma frota de equipamentos de carga, os quais devem ser alocados de acordo com suas disponibilidades de operação e produtividade. Essas questões são abordadas por Pinto e Merschmann (2001), Merschmann (2002) e Pinto et al. (2003) e definem que o ritmo de lavra de cada frente depende do equipamento de carga a ela alocada. A produtividade do equipamento de carga é limitada, superiormente, pelo número máximo de ciclos de carga por hora vezes sua capacidade de carga e, inferiormente, por um valor que torna viável sua utilização.

\subsection{Alocação estática de caminhões}

O sistema de alocação de caminhões é geralmente adotado por minera- 
ções de pequeno e médio porte, devido à simplificação das operações e ao alto custo de se implantar um sistema de despacho eletrônico de caminhões. Nesse sistema, cada caminhão é alocado a uma única rota, ou seja, permanece se deslocando entre dois pontos fixos, um de carga e outro de descarga. Os caminhões devem ser alocados a um ponto de carga que possua um equipamento de carga compatível. Essa compatibilidade está relacionada, principalmente, com o número de passes necessários para encher a caçamba do caminhão. Poucos passes podem danificar a estrutura do caminhão, enquanto muitos passes acarretam um maior tempo de carga.

\section{Modelo matemático}

O problema abordado envolve um conjunto de frentes de minério e estéril que devem ser lavradas de modo a atender as metas de qualidade e produção preestabelecidas, além de uma relação estéril/minério desejada. Existe, ainda, uma frota de equipamentos de carga, os quais devem ser alocados às frentes de modo a viabilizar a lavra. O material retirado das frentes deve ser transportado por uma frota heterogênea de caminhões, ou seja, caminhões com capacidades de transporte diferentes, os quais podem ou não ser compatíveis com determinado equipamento de carga. Essa restrição proposta por Pinto e Merschmann (2001) e também usada em Merschmann (2002) e Pinto et al. (2003) foi excluída e contemplada através de uma modelagem mais eficiente, eliminando, com isso, um número considerável de restrições no modelo, mais precisamente, $|\mathrm{F}| \mathrm{x}|\mathrm{C}| \mathrm{x}|\mathrm{V}|$ restrições, sendo $|\mathrm{F}|$ o número de frentes, $|\mathrm{C}|$ o número de equipamentos de carga e $|\mathrm{V}|$ o número de caminhões.

Para o desenvolvimento do modelo de programação matemática relativo a esse problema são definidos os seguintes dados de entrada:

$M$ : Conjunto de frentes de minério.

$E$ : Conjunto de frentes de estéril.

$F$ : Conjunto de frentes formado por $M \cup E$.
$S$ : Conjunto dos parâmetros de qualidade analisados no minério.

$C$ : Conjunto de equipamentos de carga.

$V$ : Conjunto de equipamentos de transporte.

$e_{i}$ : Economia obtida, por tonelada/hora, com a utilização do minério proveniente da frente $i$.

Pr: Ritmo de lavra recomendado ( $\mathrm{t} / \mathrm{h})$.

$P l$ : Ritmo de lavra mínimo (t/h).

$P u$ : Ritmo de lavra máximo (t/h).

$\beta$ : Penalidade por desvio negativo do ritmo de lavra.

$\beta^{+}$: Penalidade por desvio positivo do ritmo de lavra.

$t_{i j}$ : Teor do parâmetro $j$ na frente $i(\%)$.

$t_{r j}:$ Teor recomendado para o parâmetro $j$ no produto final (\%).

$t_{l j}$ : Teor mínimo admissível para o parâmetro $j$ no produto final (\%).

$t_{u j}$ : Teor máximo admissível para o parâmetro $j$ no produto final (\%).

$\alpha_{j}$ : Penalidade por desvio negativo para o parâmetro j no produto final.

$\alpha_{j}^{+}$: Penalidade por desvio positivo para o parâmetro j no produto final.

rem: Relação estério/minério mínima requerida.

$C l_{k}$ : Produção mínima do equipamento de carga $k(\mathrm{t} / \mathrm{h})$.

$C s_{k}$ : Produção máxima do equipamento de carga $k(\mathrm{t} / \mathrm{h})$.

$g_{l k}: 1$ se o caminhão lé compatível com o equipamento de carga $k$. 0 caso contrário.

$V u_{i l}:$ Produção máxima do caminhão 1 alocado à frente $i(\mathrm{t} / \mathrm{h})$.

cap: Capacidade do caminhão 1, em toneladas.

$u_{i}$ : Número máximo de caminhões que operam na frente $i$.

$t t_{i}$ : Tempo total de ciclo, em minutos, dos caminhões na frente $i$.

$t c_{i}$ : Tempo de carga, em minutos, dos caminhões na frente $i$.

Definem-se, ainda, as seguintes variáveis de decisão:

$x_{i}$ : Ritmo de lavra da frente $i(\mathrm{t} / \mathrm{h})$;

$y_{i k}: 1$ se o equipamento de carga $k$ opera na frente $i .0$ caso contrário.

$z_{i l}: 1$ se o caminhão 1 opera na frente $i .0$ caso contrário.

Definem-se, também, as seguintes variáveis de desvio:

$d_{j}$ : Desvio negativo do parâmetro $j$ no produto final $(\mathrm{t} / \mathrm{h})$.

$d_{j}^{+}$: Desvio positivo do parâmetro $j$ no produto final $(\mathrm{t} / \mathrm{h})$.

$P$ : Desvio negativo do ritmo de lavra em relação ao recomendado $(\mathrm{t} / \mathrm{h})$.

$P^{+}$: Desvio positivo do ritmo de lavra em relação ao recomendado $(\mathrm{t} / \mathrm{h})$.

O modelo de programação matemática relativo à alocação estática de uma frota heterogênea de caminhões, levando-se em consideração metas de produção e qualidade dos minérios, é apresentado pelas equações (1) a (19).

$$
\max \sum_{i \in M} e_{i} x_{i}-\sum_{j \alpha S} \alpha_{j}^{-} d_{j}^{-}-\sum_{j \alpha S} \alpha_{j}^{+} d_{j}^{+}-\beta^{-} P^{-}-\beta^{+} P^{+}
$$

$\sum_{i \in M}\left(t_{i j}-t u_{j}\right) x_{i} \leq 0 \quad \forall j \in S$ 


$$
\begin{aligned}
& \sum_{i \in M}\left(t_{i j}-t l_{j}\right) x_{i} \geq 0 \quad \forall j \in S \\
& \sum_{i \in M}\left(t_{i j}-t r_{j}\right) x_{i}+d_{j}^{-}-d_{j}^{+}=0 \quad \forall j \in S \\
& \sum_{i \in M} x_{i}-P u \leq 0 \\
& \sum_{i \in M} x_{i}-P l \geq 0 \\
& \sum_{i \in M} x_{i}-\operatorname{Pr}+P^{-}-P^{+}=0 \\
& x_{i} \geq 0 \quad \forall i \in F \\
& \sum_{i \in E} x_{i}-\operatorname{rem} \sum_{i \in M} x_{i} \geq 0 \\
& \sum_{k \in C} y_{i k} \leq 1 \quad \forall i \in F \\
& \sum_{i \in F} y_{i k} \leq 1 \quad \forall k \in C \\
& y_{i k} \in\{0,1\} \quad \forall i \in F, k \in C \\
& x_{i}-\sum_{k \in C} C s_{k} y_{i k} \leq 0 \quad \forall i \in F \\
& x_{i}-\sum_{k \in C} C l_{k} y_{i k} \geq 0 \quad \forall i \in F \\
& \sum_{i \in F} z_{i l} \leq 1 \quad \forall l \in V \\
& z_{i l}-\sum_{k \in C} y_{i k} \leq 0 \quad \forall i \in F, l \in V, g_{l k} \neq 0 \\
& x_{i}-\sum_{l \in V} V u_{i l} z_{i l} \leq 0 \quad \forall i \in F \\
& \sum_{l \in V} z_{i l}-u_{i} \leq 0 \quad \forall i \in F \\
& z_{i l} \in\{0,1\} \quad \forall i \in F, l \in V
\end{aligned}
$$

A produção máxima, em toneladas por hora, do veículo $l$ na frente $i$ e o número máximo de caminhões por frente são calculados, respectivamente, pelas equações (20) e (21).

$$
\begin{aligned}
& V u_{i l}=\frac{60 \times c a p_{l}}{t t_{i}} \quad \forall i \in F, l \in V \\
& \text { ui }=\frac{t t_{i}}{t c_{i}} \quad \forall i \in F
\end{aligned}
$$

As restrições (2) e (3) definem limites máximos e mínimos para os parâmetros de qualidade da mistura, enquanto as restrições (5) e (6) limitam o máximo e o mínimo de minério a ser produzido.

As restrições (4) e (7), propostas por Chanda e Dagdelen (1995) e desenvolvidas a partir do método de programação por metas (goal programming), visam a medir os desvios de qualidade e produção, respectivamente, em relação aos valores requeridos. A restrição (8) impede que valores negativos de produção sejam aceitos. Com a inclusão das restrições (4) e (7), a função com o único objetivo de maximizar a produção de minério utilizada em Pinto e Merschmann (2001), Merschmann (2002) e Pinto et al. (2003) foi alterada para a função multiobjetivo (1), onde deseja-se, além da maximização da produção, que sejam minimizados os desvios de produção e qualidade em relação aos valores requeridos.

A restrição (9) diz respeito ao atendimento da relação estéril/minério. A restrição (10) define que cada frente possui um único equipamento de carga, enquanto a restrição (11) define que cada equipamento de carga opera em uma única frente. A restrição (12) define se um equipamento de carga deve ou não ser alocado a uma determinada frente de lavra. As restrições (13) e (14) limitam o ritmo de lavra da frente onde um determinado equipamento de carga está alocado, de acordo com sua capacidade de produção máximas e mínimas. A restrição (15) caracteriza o problema de alocação estática de caminhões, definindo que um caminhão somente pode operar em uma única frente, onde deve estar alocado um equipamento de carga compatível (restrição (16)). A restrição (17) limita o ritmo de lavra de uma frente pela capacidade máxima de transporte dos caminhões nela alocados. A restrição (18) limita o número máximo de caminhões alocados a uma determinada frente, de forma a prevenir a formação de filas. A restrição (19) define em qual frente de lavra cada caminhão deve ser alocado.

\section{Resultados}

O modelo de programação matemática desenvolvido foi implementado no aplicativo de otimização LINGO 7.0, interfaceando com planilhas do EXCEL 2000.

Para testar o modelo, foram utilizados os dados considerados em Pinto et al. (2003). Os parâmetros de qualidade analisados referem-se aos teores de 10 elementos químicos presentes em 12 frentes de minério de um total de 17 frentes. Como o modelo de Pinto et al. (2003) não possuía metas de qualidade e produção, estas foram estipuladas como sendo a média entre os limites superior e inferior de cada um desses parâmetros. 
Os testes foram realizados em um microcomputador com processador AMD Duron $1.0 \mathrm{GHz}$ com $256 \mathrm{MB}$ de RAM, demandando, aproximadamente, 1 minuto e 15 segundos de processamento para se obter a solução ótima para o problema.

Não houve desvios de qualidade e produção em relação às metas requeridas, obtendo-se uma relação estéril/ minério de 0,3 . Foram alocados todos os 8 equipamentos de carga disponíveis, operando com produção máxima, e utilizados 23 caminhões dos 30 disponíveis, sendo 12 de 50 t e 11 de 80 t, com uma produtividade de $80,4 \%$. Em relação ao trabalho de Pinto et al. (2003), o número total de restrições utilizadas reduziu de 4751 para apenas 671 , mesmo considerando que o modelo proposto inclui outras restrições além daquelas abordadas no referido trabalho. Observa-se, também, que, para se alcançarem as metas de produção e de qualidade, foi necessário um caminhão a mais, acarretando uma redução de produtividade de 5,85\%, tomando como base a produtividade de $85,4 \%$ obtida no trabalho de Pinto et al. (2003).

\section{Conclusões}

A aplicação de métodos de pesquisa operacional na resolução de problemas de planejamento de produção em mineração é de grande importância, auxiliando a tomada de decisão de forma eficiente.

O modelo de programação linear por metas proposto nesse trabalho demonstra que é possível atingir as metas requeridas e otimizar as operações de transporte e carregamento, com uma pequena redução de produtividade. Observa-se, ademais, que esse trabalho contribui com a automação e otimização do planejamento de lavra, possibilitando às empresas de mineração uma melhor utilização de seus recursos.

\section{Referências} bibliográficas

CHANDA, E. K. C., DAGDELEN, KADRI. Optimal blending of mine production using goal programming and interactive graphics systems. International Journal of Surface Mining, Reclamation and Environment. v. 9, p. 203-208, 1995.

MERSCHMANN, L. H. C. Desenvolvimento de um sistema de otimização e simulação para análise de cenários de produção em minas a céu aberto. Rio de Janeiro: Programa de Engenharia de Produção/ COPPE/UFRJ, 2002. (Dissertação de mestrado).

PINTO, L. R., BIAJOLI, F. L., MINE, O. M. Uso de otimizador em planilhas eletrônicas para auxílio ao planejamento de lavra. Ouro Preto: Programa de Pós-graduação em Engenharia Mineral, Universidade Federal de Ouro Preto, 2003. (Relatório técnico).

PINTO, L. R., MERSCHMANN, L. H. C. Planejamento operacional da lavra de mina usando modelos matemáticos. REM Revista Escola de Minas. v. 54, n. 3, p. 211-214, 2001.

Artigo recebido em 30/06/2004 e aprovado em 23/12/2004.

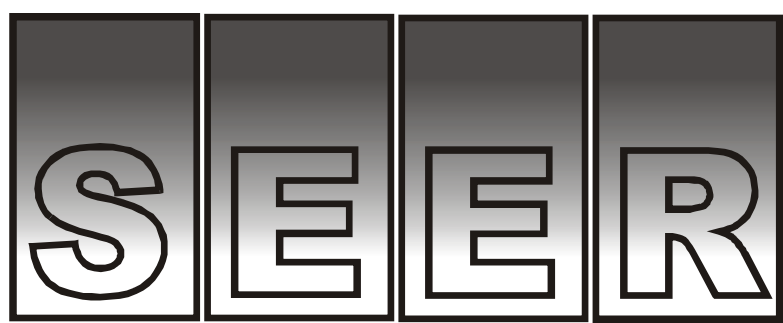

SISTEMA ELETRÔNICO DE EDITORAÇÃO DE REVISTAS
A questão SEER ou não SEER deixou de existir:

\section{Agora os autores utilizarão \\ o SEER \\ para enviar e acompanhar \\ seu artigo até o momento \\ da publicação.}

\section{REM 69 anos divulgando Ciência}

